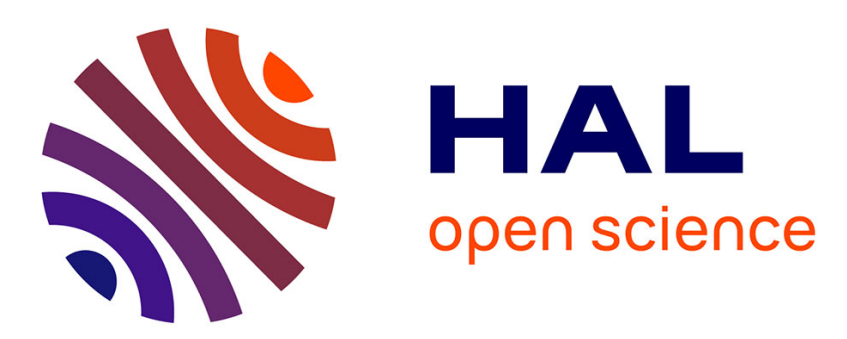

\title{
La perception de la menace communiste, en France et en Italie au début de la Guerre Froide \\ Pascal Girard
}

\section{To cite this version:}

Pascal Girard. La perception de la menace communiste, en France et en Italie au début de la Guerre Froide. Sous la direction de Philippe Buton, Olivier Büttner, Michel Hastings. La Guerre froide vue d'en bas, CNRS Editions, pp.41-53, 2014, 9782271078230. halshs-01257814

\section{HAL Id: halshs-01257814 https://shs.hal.science/halshs-01257814}

Submitted on 18 Jan 2016

HAL is a multi-disciplinary open access archive for the deposit and dissemination of scientific research documents, whether they are published or not. The documents may come from teaching and research institutions in France or abroad, or from public or private research centers.
L'archive ouverte pluridisciplinaire HAL, est destinée au dépôt et à la diffusion de documents scientifiques de niveau recherche, publiés ou non, émanant des établissements d'enseignement et de recherche français ou étrangers, des laboratoires publics ou privés. 


\section{La perception de la menace communiste, en France et en Italie au début de la Guerre froide}

Pascal Girard

La perception de la menace communiste n'est pas uniquement un objet d'histoire culturelle propre à nous replonger dans l'atmosphère de la Guerre froide. La représentation de cette menace constituait à l'époque un des guides de l'action des gouvernements, des partis politiques ou des états-majors, et en cela sa connaissance est fondamentale pour l'histoire politique de cette période.

Toutefois, cerner des perceptions n'est pas tâche aisée. Elles passent évidemment par de multiples filtres et sont conditionnées par les représentations préalables des individus. Certes, le chercheur dispose d'une masse d'archives virtuellement inépuisable; nous avons ainsi pu nous appuyer sur les archives civiles (rapport de diverses polices, rapports de préfets, dossiers d'enquêtes judiciaires, débats gouvernementaux ou ministériels), militaires (notes de renseignements, rapports d'activité, synthèses à destination de l'État-Major, dossiers d'exercices), privées (propagande et procès-verbaux des débats des partis politiques) ou encore des témoignages écrits et des entretiens.

Reste que la propagation de nouvelles, d'informations ne prouve pas nécessairement que leurs relais, comme leurs destinataires, y ont jamais cru. Les opinions n'étant que rarement exprimées, plutôt que dans les informations véhiculées, ce sont souvent les actes ou les réactions qu'il faut analyser pour tenter de trouver les preuves de la perception d'une menace. Mais même ainsi, on ne peut retirer des documents au mieux que des indices ou des éléments de preuves très partiels renvoyant à des perceptions fragmentées, atomisées, aussi variées que les institutions et les individus concernés. Si, pour la brièveté et la clarté de l'exposé, nous devrons aller à l'essentiel, il conviendra néanmoins de demeurer modeste et nuancé sur les conclusions à tirer de notre travail.

Dans ces conditions, pourquoi accentuer la difficulté en adoptant une perspective comparatiste ? De fait, au début de la Guerre froide, la France et 
l'Italie sont les pays qui comptent les deux plus puissants partis communistes d'Europe de l'Ouest. Au-delà de ce qui les oppose ${ }^{1}$, la nature des institutions (deux républiques naissantes ou renaissantes), la configuration politique (des gouvernements de centre dont les communistes sont exclus à partir de mai 1947) et la situation du maintien de l'ordre (avec des effectifs policiers et militaires insuffisants) justifient une comparaison qui sera ici destinée à mettre avant tout en évidence les caractéristiques de la situation française.

Cette approche comparatiste a toutefois pour inconvénient d'élargir le champ d'étude et de nous condamner, dans le cadre de cette publication, à être encore plus général dans nos analyses. C'est pourquoi nous nous concentrerons sur une période assez brève, celle des débuts (et en même temps de l'apogée) de la Guerre froide, de 1947 à 1953. De plus, sur le détail des représentations de la menace communiste en France, en particulier au sein de l'armée, ou sur la question de la répression étatique à l'encontre du Parti communiste, nous renvoyons d'une part aux communications d'Annie Martin et Olivier Büttner et d'Alain Bancaud respectivement, et d'autre part aux chapitres consacrés à ces questions dans notre doctorat ${ }^{2}$. Nous pourrons ainsi nous concentrer sur le contraste entre les situations française et italienne puis sur les facteurs qui pourraient l'expliquer.

\section{L’IMPACT DES VIOLENCES SUR LA PERCEPTION DES PARTIS COMMUNISTES}

Dans les deux pays, l'automne 1947 constitue le tournant en ce qui concerne la perception d'une menace communiste potentielle, qui passe de la peur des grèves ou des manifestations d'ampleur et de gravité limitées à celle d'actes de subversion et d'insurrection, voire d'un coup d'État ou d'une révolution. Ce changement est évidemment lié à l'entrée dans la Guerre froide, elle-même marquée par la conférence de Szklarska-Poreba, mais cette inflexion n'est pas nécessairement claire pour l'opinion, les autorités civiles et militaires, ou même les militants communistes.

Ce qui matérialise cette rupture et transforme la nature de la menace communiste supposée, c’est une vague inédite de violences qui touche les

1. Lazar Marc, Maisons rouges. Les partis communistes français et italien de la Libération à nos jours, Paris, Aubier, 1992, en particulier p. 27.

2. La plus grande partie de ce développement est tiré de notre travail : Girard Pascal, Les complots politiques en France et en Italie, de la fin de la Seconde Guerre mondiale à la fin des années 1950, Thèse (dir. : Heinz-Gerhardt Haupt), Florence, Institut Universitaire Européen, 2012 (p. 305-697 pour l'essentiel). 
deux pays presque simultanément (en octobre-novembre pour l'Italie et en novembre-décembre pour la France), et qui résulte d'un vaste mouvement de grève impulsé par les partis communistes et leurs syndicats respectifs (CGT et CGIL).

Ces violences sont autant physiques (contre les policiers ou les « jaunes », les non-grévistes) que matérielles (sabotages d'outil de production, de moyens de transport ou de voies de communication) et verbales. Elles accompagnent les manifestations, les occupations d'usines ou encore les barrages routiers et ferroviaires ; elles peuvent être meurtrières, et dénotent assez souvent une préparation préalable de la part des militants communistes qui y participent (stratégie, armes par destination) ${ }^{3}$. L'impact de ces violences est renforcé par une seconde vague d'affrontements plus brève, mais encore plus violente, en particulier en Italie : ce sont en juillet 1948 les émeutes consécutives à la tentative d'assassinat contre Palmiro Togliatti, secrétaire général du PCI, et en octobre-novembre 1948, les grèves dans les bassins miniers français.

La conséquence de ces violences est qu'à partir de l'automne 1947, on constate une double évolution dans les rapports contenus dans les archives de police des deux pays. C'est d'abord un afflux de nouvelles très alarmistes faisant état de préparatifs insurrectionnels de la part du Parti communiste et de ses organisations satellites. Ces nouvelles s'apparentent le plus souvent à des rumeurs ou des révélations d'informateurs non identifiés, et il est donc impossible de les exploiter telles quelles afin d'évaluer la prégnance de la peur d'un coup de force communiste. En effet, elles peuvent aussi bien être l'effet des craintes d'une partie de la population que des manipulations justement destinées à inquiéter et influencer les forces de police, et au-delà, le gouvernement. Reste que, et c'est le second point, plus décisif, les autorités civiles et militaires semblent prendre cette accumulation d'informations au sérieux. Les services de police notamment mènent des enquêtes au sujet d'informations inquiétantes dont la crédibilité est parfois très faible. En tout état de cause, cela démontre que dans l'esprit d'un certain nombre de fonctionnaires de police et de leurs supérieurs, le risque existe et qu'il serait imprudent de le négliger. Élément révélateur de cet état d'esprit nouveau provoqué par les violences, les ministres de l'Intérieur eux-mêmes ordonnent des enquêtes sur la question. En décembre 1947, Jules Moch demande aux IGAME, et à travers eux aux préfets, d'élaborer une synthèse sur les grèves qui viennent de se dérouler

3. Sur ce point, nous renvoyons à Girard Pascal, «La violence militante communiste à l'apogée de la guerre froide », in Audigier François, Girard Pascal (dir.), Se battre pour ses idées. La violence militante en France des années 1920 aux années 1970, Paris, Riveneuve, 2011, p. 93-117. 
avec, parmi les préoccupations centrales, l'objectif de déterminer s'il s'agissait d'un mouvement insurrectionnel ${ }^{4}$. De même, le ministre de l'Intérieur italien, Mario Scelba, à la suite des émeutes meurtrières de juillet 1948, demande aux préfets de transmettre des rapports afin de déterminer si les violences ont été la conséquence d'un « plan général » 5 .

Toutefois, imputer aux seules violences cette forte croissance de la perception du péril par les autorités civiles et militaires serait spécieux. En effet, la menace communiste intérieure ne peut se séparer d'une autre menace communiste, celle venue de l'autre côté du rideau de fer.

\section{L’OMBRE PORTÉE DE L’AFFRONTEMENT EST-OUEST}

Il est difficile d'évaluer l'impact de la peur de l'expansionnisme soviétique, que ce soit lors du coup de Prague ou du blocus de Berlin, sur un temps relativement court. Mais le lien entre menace communiste nationale et péril extérieur soviétique est mis en lumière par la chronologie du second temps fort de la peur du coup de force communiste. En effet, l'année 1949, relativement plus apaisée dans les deux pays (même si la question agraire entretient un climat plus agité et violent en Italie), conduit à un fléchissement du nombre et de la gravité des rapports et des informations concernant le danger communiste (les inquiétudes portant essentiellement sur des grèves violentes et localisées). Mais l'été 1950 entraîne un nouveau pic des craintes.

Celui-ci peut certes être mis en rapport avec un certain regain des violences au début de l'année 1950, lié à la lutte contre la livraison d'armes américaines $^{6}$, mais le facteur principal est bien évidemment le déclenchement de la guerre de Corée le 25 juin. Le contexte d'une guerre mondiale à venir $^{7}$ rend particulièrement aiguë la crainte de la $5^{\mathrm{e}}$ colonne communiste,

4. Demande de renseignements, 12 décembre 1947, Archives Départementales de Haute-Garonne, 1960 W 68.

5. Circulaire, 19 juillet 1948, Archivio Centrale di Stato (ACS), Ministero dell'Interno (MI), Direzione Generale della Pubblica Sicurezza (DGPS), Affari Generali e Riservati, 1947-1948, busta 120.

6. Guiso Andrea, La colomba e la spada. « Lotta per la pace » e antiamericanismo nella politica del partito comunista italiano (1949-1954), Soveria Mannelli, Rubbettino Editore, 2006, p. 242-248 ; Ruscio Alain, Les communistes français et la guerre d'Indochine, 1944-1954, Paris, L'Harmattan, 1985, p. 251-258.

7. Sondages, $\mathrm{n}^{\circ} 1$, 1951, p. 8-10 et Soutou Georges-Henri, « La perception de la menace soviétique par les décideurs de l'Europe occidentale : le cas de la France », in Dockrill Saki, Frank Robert, Soutou Georges-Henri, Varsori Antonio (dir.), L'Europe de l'Est et de l'Ouest dans la Guerre Froide, 1948-1953, Paris, PUPS, 2002, p. 30-34. 
c'est-à-dire d'un parti communiste qui minerait de l'intérieur les capacités de défense nationale afin d'aider les troupes du bloc de l'Est qui envahiraient au même moment le pays. Autrement dit, le Parti communiste est soupçonné tant en France qu'en Italie d'être prêt à se livrer à la trahison en temps de guerre, si besoin les armes à la main.

Cette peur, dont rien ne dit en l'état qu'elle ait été fondée, est attisée par le Parti communiste lui-même, en particulier en France ${ }^{8}$. À partir de l'automne $1948^{9}$ et jusqu'au début de l'année 1949, le Bureau politique du PCF comme les dirigeants les plus importants (Jacques Duclos puis Maurice Thorez) multiplient les déclarations laissant penser qu'en cas de guerre, les militants communistes combattraient aux côtés de l'envahisseur soviétique ${ }^{10}$. Au soir de la publication de la déclaration de Maurice Thorez dans L'Humanité du 22 février 1949, un vif débat s'engage à l'Assemblée nationale. Toutefois, en dépit des appels de certains députés gaullistes à poursuivre judiciairement les dirigeants du PCF, c'est un ordre du jour se bornant à condamner moralement les propos de Thorez qui est finalement voté par les députés de la Troisième Force, modérés compris ${ }^{11}$.

Avec le commencement de la guerre de Corée, l'appréhension beaucoup plus dramatique du danger se traduit à la tête des deux États par des projets de loi très comparables. En France, il s'agit du projet de « défense en surface ", préparé par le ministre de la Défense nationale Jules Moch, rapidement approuvé en Conseil des ministres et traduit par le décret du 29 septembre 1950 présenté devant les députés le 26 octobre suivant. Le but de cette organisation est d' « assurer la sécurité des communications, lutter contre les éléments extérieurs parachutés, débarqués ou infiltrés, s'opposer à toute tentative de sabotage, assurer le maintien de l'ordre ». Cette « défense en surface » vise, en cas de mobilisation, mais aussi de situation menaçante, à assurer le maintien de l'ordre intérieur grâce à la coordination entre les forces armées, la gendarmerie, la police et une future "garde territoriale » de réservistes et de volontaires. Même si la menace commu-

8. On trouve des déclarations du même ordre en Italie, mais par des orateurs locaux. Voir notamment les rapports des 23 mars, 24 décembre 1949 et 27 janvier 1950 dans ACS, MI, DGPS, Gabinetto, Partiti Politici, 1944-1966, busta 45.

9. «Le peuple de France ne fera jamais la guerre à l’Union soviétique ", L'Humanité, 5 novembre 1948.

10. Sur ce point, voir la synthèse de Santamaria Yves, Le Parti de l'ennemi ? Le Parti communiste français dans la lutte pour la paix (1947-1958), Paris, Armand Colin, 2006, p. 81-100. Ces propos sont repris dans la propagande communiste locale - voir les notes d'information, 23 février et 11 mars 1949, Archives de la Préfecture de Police (APP), BA 1883.

11. Journal Officiel, Débats, Assemblée Nationale, 24.2.1949, p. 885-900 en particulier. 
niste n'est jamais explicitement citée, les discours de Jules Moch à l'Assemblée nationale des 28 juillet et 26 octobre 1950 montrent que c'est bien l'action de sabotage d'une 5e colonne qui est visée ${ }^{12}$. En Italie, au cours de l'été 1950, Mario Scelba met lui sur pieds un projet de « défense civile », présenté à la Chambre des députés le 14 octobre 1950 et destiné à couvrir les arrières de l'armée en cas de situation grave, grâce notamment à l’organisation de corps auxiliaires composés de civils.

Certes, ces deux projets seront des échecs. Le projet de loi de « défense civile », voté en juillet 1951 par les députés italiens, ne sera jamais examiné par le Sénat ${ }^{13}$. Quant à la " défense en surface » française, si elle a fait l'objet d'un décret, elle ne sera jamais mise en œuvre sur le terrain à cause de difficultés d'ordre juridique et du mauvais vouloir de l'ÉtatMajor. Ce rapide désintérêt est d'ailleurs la preuve a contrario que c'est bien la guerre de Corée qui avait aiguillonné les craintes à l'encontre de la $5^{\mathrm{e}}$ colonne. En effet, comme le relève fort justement Isabelle Miclot dans son article (infra), passé l'automne 1950, la peur d'une généralisation du conflit coréen décroît rapidement - c'est ce que démontre notamment le climat relativement apaisé dans lequel se déroulent les élections de juin 1951 en Italie et en France.

\section{L'ÉTRANGER SUBVERSIF}

Un dernier point commun entre les deux pays est que l'association entre ces deux menaces intérieure et extérieure conduit les autorités civiles et militaires à considérer le rôle des étrangers comme décisif dans les menées violentes, subversives et potentiellement insurrectionnelles des organisations communistes.

En France, la méfiance des autorités est par exemple dirigée contre les ressortissants et les associations des pays de l'Est; une instruction pour atteinte à la Sûreté extérieure de l'État aboutit en 1949 à la perquisition des locaux de diverses associations polonaises, puis en janvier 1950 à la dissolution de ces associations et à l'expulsion de certains de leurs membres ${ }^{14}$.

12. Villatoux Marie-Catherine, La défense en surface (1945-1962). Le contrôle territorial dans la pensée stratégique française d'après-guerre, Paris, Service Historique de la Défense, 2009, p. 160-162.

13. Sur cette question, voir en particulier Mazzei Federico, De Gasperi e lo « Stato Forte ». Legislazione antitotalitaria e difesa della democrazia negli anni del centrismo (1950-1952), Florence, Le Monnier-Mondadori, 2013, p. 85-110.

14. Sur cette affaire, cf. Service Historique de l'Armée de Terre (SHAT), 1 R 16, dossier 3. 
On note une même vigilance vis-à-vis des associations yougoslaves ou italiennes, qui sont surveillées et éventuellement dissoutes. Mais ce sont surtout et avant tout les Espagnols qui focalisent l'attention des autorités françaises. En effet, après la guerre civile, nombre de guérilleros ont trouvé refuge de l'autre côté des Pyrénées avec pour objectif de poursuivre la lutte contre le franquisme, et si tous ne sont pas communistes, certains ont participé aux maquis de la Résistance FTP. Les récriminations comme les inquiétudes à l'encontre des combattants antifranquistes se font jour dès la Libération, mais avec l'entrée dans la Guerre froide et les grands mouvements de grève, les rapports se succèdent afin de connaître l'organisation, les effectifs, l'équipement et plus encore les objectifs de guérilleros soupçonnés de constituer les futures « Brigades Internationales » d'une insurrection communiste en France ${ }^{15}$. La série de rapports transmis par les préfets au ministre de l'Intérieur au printemps 1950, l'inquiétude du directeur de la Sûreté Nationale puis le déclenchement de la guerre de Corée amènent à l'exécution de l'opération opportunément nommée « Boléro-Paprika » de la nuit du 7 au 8 septembre 1950 au cours de laquelle près de trois cents militants communistes étrangers, dont une majorité d'Espagnols, sont arrêtés.

Les autorités civiles finiront par convenir du caractère inoffensif d'organisations espagnoles qu'elles ont par ailleurs décapitées, mais les archives militaires témoignent de la persistance de ce type de représentations. L'épais rapport du contrôleur général de l’Armée de 1953 prévoit encore que les étrangers assumeraient un rôle essentiel de $5^{\mathrm{e}}$ colonne en cas d'invasion soviétique : un vingtième d'entre eux y prendrait part tandis que seulement un trentième des militants communistes serait impliqué. Alors même que la guerre d'Algérie n'a pas commencé, ce même rapport prévoit que pas moins de la moitié des Nord-Africains présents en métropole seraient recrutés par «l'armée populaire» insurrectionnelle ${ }^{16}$. Pour l'exercice militaire "Aquitaine » des régions militaires de Bordeaux et Poitiers de l'automne 1957, ce sont encore les guérilleros espagnols qui sont censés jouer un rôle déterminant dans les maquis intérieurs de la $5^{\mathrm{e}}$ colonne communiste, avec le secours des Nord-Africains (cependant divisés entre partisans du FLN et du MNA) ${ }^{17}$.

15. Outre Dulphy Anne, «À l'épreuve de la guerre froide : les préfets du SudOuest et les réfugiés espagnols, 1947-1953 », in Vaïsse Maurice (dir.), Les préfets, leur rôle, leur action dans le domaine de la Défense de 1800 à nos jours, ParisBruxelles, LGDJ-Bruylant, 2001, p. 353-371, voir les rapports de 1947-1948 dans APP, BA 2119 et 2157 et Archives nationales (AN), 19960325/4 ; pour 1948-1950, AN, F7 15589 et AN, 19960325/1.

16. Rapport du Contrôleur Général de l’Armée Libermann, 15 juillet 1953, SHAT, 9 R 164, dossier 1.

17. Exercice « Aquitaine », 30 septembre-5 octobre 1957, AN, 19770120/25. 
En Italie, les étrangers qui sont désignés par d'innombrables rapports et informateurs comme les instigateurs d'une insurrection et les animateurs d'une possible $5^{\mathrm{e}}$ colonne, sont quasi systématiquement des Slaves, avec un partage des tâches relativement bien défini. Les Soviétiques (des généraux ou des personnels d'ambassade) planifient et supervisent les opérations tandis que les Yougoslaves préparent l'action depuis leur base-arrière puis la dirigent sur le terrain, les communistes italiens servant au mieux de piétaille. Le rôle privilégié supposé des Yougoslaves dans la menace communiste ne s'arrête pas immédiatement avec la rupture géopolitique entre la Yougoslavie titiste et l'URSS, rendue officielle en juillet 1948 (et parfois considérée comme une ruse), mais il recule logiquement au profit d'autres Slaves, opérant cette fois depuis la Tchécoslovaquie. Si les rumeurs de putsch préparé depuis ce pays au début des années 1950 n’apparaissent pas plus crédibles que celles concernant la Yougoslavie, elles peuvent tout de même s'appuyer sur le fait (très documenté désormais ${ }^{18}$ ) que la Tchécoslovaquie sert alors de refuge à des militants communistes italiens poursuivis par la justice.

Au final, des deux côtés des Alpes, le Parti communiste est, suivant le contexte, soupçonné de vouloir semer le désordre, fomenter un coup d'État, voire de commettre la pire des trahisons, mais il semble somme toute difficilement concevable pour les autorités civiles et militaires que les militants communistes dans leur ensemble puissent être des traîtres; ce sont donc les étrangers, les " sans-patrie » et autres éléments " allogènes » qui constitueraient la menace réelle et concrète sur le terrain. Toutefois, audelà de ces points communs, la comparaison entre les deux pays met aussi en évidence des divergences profondes, qu'il s'agit d'examiner.

\section{UNE MENACE PLUS FORTEMENT RESSENTIE EN ITALIE}

Même s'il faut se défier d'un possible effet de sources, le travail d'archives fait apparaître un contraste frappant: que ce soit au sein des administrations civiles et militaires, dans les rapports de police, et de ce que nous pouvons savoir de l'opinion, la perception de la menace communiste apparaît à la fois comme plus précoce, plus intense et plus durable en Italie qu'en France. Les éléments de preuves sont nombreux.

18. Notamment grâce aux parcours racontés dans Testa Ludovico, «La vita è lotta ». Storia di un comunista emiliano, Reggio Emilia, Diabasis, 2007, p. 179 sq. et Fiori Giuseppe, Uomini ex. Lo strano destino di un gruppo di comunisti italiani, Turin, Einaudi, 1993, p. 11 sq. 
C'est d'abord l'accumulation bien plus grande en Italie de rapports alarmistes de la police et des carabinieri, qui répercutent parfois les nouvelles les plus fantaisistes sans aucun esprit critique auprès des ministères des Armées et de l'Intérieur. Ce sont, dans le même ordre d'idée, les enquêtes déclenchées en retour, parfois sur l'ensemble du territoire national, à la diffusion de la moindre rumeur de coup d'État, d'attentat ou de menées subversives, comme pour les cérémonies de l'Année sainte à Rome ${ }^{19}$, ou sur la base d'informations qui sont manifestement des provocations ${ }^{20}$.

Or, en comparaison, en France, le nombre de rapports et d'informations confidentielles (et d'enquêtes qui en sont la conséquence) est beaucoup plus réduit. Certes, des personnalités politiques sont plus impressionnables que d'autres, au premier chef le président de la République Vincent Auriol, qui a laissé dans son Journal divers témoignages d'alarmes extrêmement vives ${ }^{21}$ (souvent utilisés par des historiens pour accréditer l'idée d'une psychose anticommuniste généralisée). On trouve également des documents de police français qui rappellent les longues listes de militants communistes rapportées de façon récurrente par la police italienne en guise d'organigramme de l'organisation secrète du $\mathrm{PCI}^{22}$. Mais dans l'ensemble, les nouvelles sont bien plus rares et leur traitement plus circonspect qu'en Italie. Pour ne prendre qu'un exemple d'importance, l'enquête (évoquée plus haut) demandée par Jules Moch en décembre 1947 débouche en avril 1948 sur un épais rapport qui, sur la base de l'examen des faits rapportés par les préfets, ne conclut nullement à une tentative d'insurrection de la part du PCF. En Italie, les rapports des préfets à Mario Scelba sur les émeutes de juillet $1948^{23}$ aboutissent quasi invariablement à la même conclusion, y compris dans des provinces (relativement nombreuses) où il ne s'est rien passé de grave : les émeutes résultaient bien d'un plan insurrectionnel préparé de longue main, conclusion d'ailleurs publiquement et au moins partiellement reprise par le ministre de l'Intérieur lui-même dans les semaines qui suivent.

Enfin, à une échelle qui serait proprement celle de « la Guerre froide vue d'en bas », alors qu'il est pratiquement impossible de trouver de tels témoignages dans l'opinion française, des indices démontrent que la peur de l'insurrection communiste peut être clairement exprimée par une frac-

19. Dossier dans ACS, MI, DGPS, Affari Riservati, 1948-1950, busta 9.

20. Pour un exemple significatif, rapport, avril 1948, Archivio Luigi Sturzo (ALS), Fondo Scelba, $2^{\circ}$ versamento, busta 24.

21. Il fait même part de ses craintes d'une $5^{\mathrm{e}}$ colonne communiste jusque durant les débats des Comité de la Défense Nationale. Procès-verbal du Comité de Défense Nationale, 24 avril 1952, AN, 552 AP 43.

22. C'est le cas de l'imposant rapport « Le PCF en 1950 », AN, 19960325/1.

23. ACS, MI, DGPS, Affari Generale e Riservati, 1947-1948, busta 120-125. 
tion de la population italienne, comme à l'automne $1947^{24}$, en liaison (autre fait proprement italien) avec les événements qui se déroulent en même temps en France ${ }^{25}$.

Cette attention portée à ce qui se passe en France est d'ailleurs partagée par les autorités civiles et les membres du gouvernement italiens, dont les inquiétudes à l'égard de la menace communiste sont si fortes qu'elles peuvent être mesurées à l'aune du vocabulaire. En septembre 1947, Mario Scelba évoque en conférence de presse l'existence d'un «plan K » visant à la prise du pouvoir par le PCI, expression qui connaît un tel succès dans les milieux anticommunistes que le ministre devra en démentir l'existence peu après les élections du 18 avril 1948. Le 11 juillet 1950, lors d'un discours de politique étrangère à la Chambre des députés, c'est le Président du Conseil Alcide De Gasperi en personne qui utilise l'expression $5^{\mathrm{e}}$ colonne, aussitôt reprise à satiété par les journaux proches du gouvernement ${ }^{26}$. Cette virulence du discours politique et gouvernemental, que l'on ne retrouve pas en France (l'expression $5^{\mathrm{e}}$ colonne n'est par exemple pas employée dans le débat politique public, sauf sur des affiches de propagande du RPF ou de Paix et Liberté), traduit sans aucun doute le fait que le péril communiste est pris plus au sérieux en Italie. Comment expliquer un tel contraste à partir de situations en apparence comparables ?

\section{HISTOIRE ET GÉOPOLITIQUE}

En fait, les similitudes du contexte de Guerre froide masquent de profondes différences, notamment en ce qui concerne les héritages historiques. Les forces politiques italiennes de centre-droit et de droite sont en effet les héritières de cultures politiques anciennes, en particulier catholique et fas-

24. On le note à plusieurs reprises dans les rapports des carabinieri de l'ensemble du territoire italien pour les mois de septembre-décembre 1947. ALS, Democrazia Cristiana, Segretaria Politica, Informazione Riservate, scatola 241.

25. Voir les propos tenus en marge d'une enquête d'opinion d'avril 1948. Luzzatto Fegiz Pierpaolo, Il volto sconosciuto. Dieci anni di sondaggi Doxa, 1946-1956, Milan, Giuffrè Editore, 1956, p. 479-481.

26. « De Gasperi si appella alla solidarità nazionale contro l'azione disagregatrice delle quinte colonne », Il Popolo, 12.7.1950 ; "Attività delle quinte colonne. Il Partito Comunista Italiano fornito di armi dalla Russia ? », Il Tempo, 2.10.1950 ; « PC e quinta colonna », Il Tempo di Milano, 15.10.1950. 
ciste $^{27}$, plus foncièrement et violemment anticommunistes que celles des droites françaises, et qui impriment par la même une défiance et une peur « structurellement » plus forte à l'encontre du communisme - en particulier au sein d'une administration largement issue du ventennio fasciste, facilement en proie au « complotisme ».

De son côté, le PCI n'est pas le PCF. Marqué par la répression, la clandestinité et l'exil, il s'est constamment construit dans la lutte, alors que les communistes français peuvent se targuer avec le Front populaire d'un passé quasi gouvernemental au cours duquel ils se sont efforcés d'apparaître (et sont au moins partiellement devenus) républicains et patriotes. Le PCI est également beaucoup plus militarisé dans son organisation que le PCF (ce qui est notable dans la mise sur pied des Brigate Garibaldine au début de l'année 1948), car plus massivement engagé dans la lutte résistante et pris dans un combat contre le fascisme qui donne aux années 1943-1945 le caractère d'une véritable guerre civile que la France n'a pas connue, du moins pas au même degré28. Si le péril communiste est souvent peint sous les traits du partigiano en Italie, le FTP, pour avoir marqué certains esprits (en particulier dans l'Armée) n'est pas une figure aussi dominante dans les représentations anticommunistes françaises. Tout cela explique que le Parti communiste italien constitue un fauteur de coup d'État beaucoup plus crédible que son homologue français.

Enfin, le cycle violences-« répression préventive ${ }^{29}$ de l'époque, qui entretient la peur de la menace communiste, est beaucoup plus intense et meurtrier en Italie qu'en France ${ }^{30}$; il renvoie certes au contexte immédiat de la Guerre froide, mais aussi et surtout à une histoire sociale et politique

27. Pertici Roberto, « Il vario anticomunismo italiano (1936-1960) : lineamenti di una storia », in Di Nucci Loreto, Galli della Loggia Ernesto, Due nazioni. Legittimazione e delegittimazione nella storia dell'Italia contemporanea, Bologne, Il Mulino, 2003, p. 263-284 ; Scoppola Pietro, « Aspetti e momenti dell'anticomunismo », in Ventrone Angelo (dir.), L'ossessione del nemico. Memorie divise nella storia della Repubblica, Rome, Donzelli Editore, 2006, p. 71-78; Lepre Aurelio, L'anticomunismo e l'antifascismo in Italia, Bologne, Il Mulino, 1997, p. 17-121.

28. Buton Philippe, «La Francia della Liberazione e la guerra civile », Memoria e Ricerca, $\mathrm{n}^{\circ}$ 21, 2006, p. 101-111.

29. Della Porta Donatella, Reiter Herbert, Polizia e protesta. L'ordine pubblico dalla liberazione ai «no global », Bologne, Il Mulino, 2003, p. 76 et 100.

30. Même si les inventaires sont nombreux et divergent en fonction des types d'affrontements pris en compte, le bilan des violences sociales et politiques est très lourd parmi les forces de l'ordre mais avant tout chez les manifestants et les grévistes ; la fourchette basse est de plusieurs dizaines de morts sur la seule période 1947-1950 et d’au moins une centaine pour les années 1947-1953. 
italienne beaucoup plus brutale. Claudio Pavone ${ }^{31}$ a souligné que la lutte résistante a été une guerre patriotique de libération nationale, mais aussi une guerre civile et une guerre de classes prolongeant toutes deux les affrontements, notamment agraires ${ }^{32}$, du début des années 1920. Si la Guerre froide semble donc plus lourde de menaces en Italie, c'est également parce qu'elle se coule dans un climat politique et social qui a eu cours tout au long du $\mathrm{Xx}^{\mathrm{e}}$ siècle et qui a été bien plus conflictuel et sanglant que celui de la France.

Il faut enfin prendre en compte la géographie de l'Italie. En effet, si les Slaves en général, et les Yougoslaves en particulier, sont aussi craints, c'est qu'au début de la Guerre froide, l'Italie est en contact direct avec le bloc de l'Est par le biais de la frontière yougoslave, et constitue donc une porte d'entrée toute désignée pour l'invasion soviétique. De plus, la Yougoslavie est associée aux exactions de la fin de la guerre (en particulier les foibe), et durant les premières années de l'après-guerre, la province de Gorizia connaît une violence intercommunautaire (à laquelle les communistes italiens participent aux côtés des philo-Slaves) particulièrement soutenue, même en la comparant aux autres provinces. Cela entretient évidemment l'image d'un Parti communiste violent et aux ordres de l'étranger, tout autant que l'idée d'une Yougoslavie responsable de menées subversives hostiles à l'Italie.

Cela n'est d'ailleurs pas sans conséquence sur le plan militaire : alors que l'hypothèse d'une évacuation du territoire national par le gouvernement en cas d'attaque soviétique n'est que brièvement évoquée en France en juin 1948, l’idée d'un repli sur le « réduit sarde » est clairement envisagée par l'État-Major italien à la même époque ${ }^{33}$.

La peur de la menace communiste résulte donc de la combinaison (complexe, comme le prouve le rôle attribué aux étrangers) de facteurs intérieurs et extérieurs, et varie sans surprise en fonction des individus, mais aussi du moment et du lieu, ce qui ne fait que rendre plus évident encore l’apport irremplaçable des études menées à l'échelle départementale.

31. Pavone Claudio, Una guerra civile. Saggio storico sulla moralità nella Resistenza, Turin, Bollati Boringhieri, 1991 (éd. fr. Le Seuil, 2005).

32. Crainz Guido, «I braccianti padani », in Chianese Gloria, Crainz Guido, Da Vela Marco, Gribaudi Gabriella, Italia 1945-1950. Conflitti e trasformazioni sociali, Milan, FrancoAngeli, 1985, p. 234-264 ; Goretti Leo, "I "neri bianchi”. Mezzadri di Greve in Chianti tra lotte sindacali e fuga dalle campagne (19451960) », Rome, Odradek, 2008, p. 39-74.

33. Ministero della Difesa, Stato Maggiore dell'Esercito, Memoria sulla Difesa dell'Italia verso Oriente e Appunto, février 1948, Ufficio Storico dell'Esercito Italiano, H-6, raccogliatore 27. 
La comparaison entre les deux pays fait apparaître une Italie travaillée par la violence, par les héritages politiques et par la situation géopolitique, et par conséquent en proie à une crainte plus grande vis-à-vis du danger communiste. Par contraste, la France est atteinte plus modérément, ce qui se traduit aussi bien dans le vocabulaire du débat politique que dans la moindre rigueur de la répression policière et judiciaire à l'encontre du PCF.

Il est toutefois absolument crucial de garder à l'esprit que ce travail est, comme l'a formulé Michel Hastings, une histoire « de la trace », "de la miette ». En effet, la masse d'archives que nous avons consultées, et qui est incommensurablement plus importante que celle que nous avons utilisées ici, n'évoque jamais une quelconque menace communiste ; celle-ci constitue à l'évidence, même durant ces années de Guerre froide, une préoccupation bien moindre que le ravitaillement ou l'État des finances publiques, tant à la tête de l'État qu'au sein des populations. De plus, cette crainte s'atténue encore avec le temps dans les deux pays, de façon particulièrement nette et sans surprise au début des années 1950, même si cela est globalement plus lent en Italie qu'en France, et plus rapide pour les autorités civiles que pour les autorités militaires (nous confirmons sur ce point également les conclusions d'Olivier Büttner et Annie Martin). Il importe donc de se défier de l'illusion de deux pays littéralement obsédés par le danger communiste pour tirer, à notre sens, deux conclusions principales de cette approche comparatiste.

La première est que l'idée d'une "peur du rouge " à l'origine d'un «maccarthysme à la française », sans doute séduisante, mais bien peu étayée sur le plan historique, est à relativiser d'autant plus fortement que le cas italien la ramène à sa juste mesure. La seconde est que le contexte de Guerre froide n'a pas d'effets mécaniques sur la vie politique, sociale et culturelle et qu'il n'agit dans ces différents domaines que par le rejeu de facteurs proprement nationaux. Si la Guerre froide semble avoir un impact plus important en Italie, c'est qu'elle se superpose à d'anciens conflits « d'en bas » auxquels elle donne une acuité nouvelle: dans une certaine mesure, au-delà des Alpes, la Guerre froide aura été la continuation de la guerre civile, mais par d'autres moyens. A contrario, et sauf ponctuellement, la Guerre froide vue de France révèle, en creux, le long processus de pacification de la vie sociale et politique opéré depuis la fin du XIX ${ }^{\mathrm{e}}$ siècle et l'avènement de la République. 